\title{
Infection Prevention and Control: A Biodefense Measure
}

\author{
Saskia Popescu
}

\subsection{Infection Prevention and Control}

Infection prevention and control is a series of practices, processes, and programs within healthcare to reduce the spread of infection among patients and staff. Infection prevention and control (IPC) programs within hospitals and healthcare facilities typically consist of infection preventionists (IPs) who are commonly nurses, epidemiologists, and microbiologists. The duties of the IP and IPC program includes a variety of responsibilities; disease surveillance, case investigation for hospitalassociated infections, education, training of staff, policy review, risk assessments for construction in patient care areas, emergency preparedness, reporting of communicable diseases to local health departments, reporting of healthcare-associated infections for reimbursement and federal requirements, etc. Traditionally, the role of the infection prevention program is to focus on reducing healthcare-associated infections (HAIs) however, communicable diseases play a large role in their duties. Properly training and educating staff on the correct use of personal protective equipment (PPE) and ensuring isolation precautions are correctly maintained is a daily responsibility for the IPC department. Aside from ensuring staff compliance to PPE and isolation precautions, IPC works to educate and facilitate compliance among visitors, as they can be a source for disease spread within a healthcare facility. The role of the IPC program and infection preventionists is also as the connective tissue within healthcare facilities to ensure that infectious diseases have the proper response across the healthcare sector, which includes departments like environmental services, security, emergency medicine, facilities, administration, interventional radiology, sterile processing departments, etc. Infection and control programs are the

S. Popescu, MPH, MA, CIC, $\mathrm{PhD}(\mathrm{c})(\bowtie)$

George Mason University, Fairfax, VA, USA

Phoenix Children's Hospital, Phoenix, AZ, USA

e-mail: spopesc2@gmu.edu 
sentinels for underlying issues within the health of the community and healthcare facility.

\subsubsection{Practitioners}

Infection prevention and control departments are comprised of infection preventionists, who have a variety of backgrounds. Traditionally the IP role has been primarily nurse-driven, but recent years have seen an influx of epidemiologists as the role increasingly involves hospital epidemiology, outbreak investigation, and public health response. The IP, even at the most novice level, must have a rudimentary understanding of microbiology, disease transmission, and public health interventions. IP competencies are built up according the Association for Professionals in Infection Prevention and Epidemiology (APIC) model, which is considered the gold standard. The APIC competency model is based upon a text that includes topics that highlight the range of IP duties - occupational health, sterilization and disinfection, bioterrorism, emergency preparedness, outbreak response, infection prevention in a variety of settings (e.g., behavioral health, correctional facilities, dental services), aseptic technique, infection prevention for specialty care populations (e.g., burns, pediatrics, organ transplants), as well as the environmental components to infection prevention work (e.g., waste management, heating and ventilation, healthcare textiles). The duties and capabilities of the IP are required to be extensive and diverse. Infection preventionists are expected to sit for the Certification in Infection Control (CIC) after 2 years. The CIC is a complex certification which aims to ensure the IP is well-versed in the variety of issues that plague healthcare systems. Since the 2002 severe acute respiratory syndrome (SARS) coronavirus and 2013-2016 Ebola virus disease outbreaks, infection preventionists' duties have increasingly involved high-consequence pathogens and emergency preparedness. Overall, infection preventionists are responsible for all manner of infectious disease threats within a healthcare facility, regardless of origin.

\subsubsection{Surveillance and Coordination with Health Departments}

Perhaps one of the most crucial responsibilities of the infection prevention and control program is to perform surveillance and report communicable diseases to local health departments. While IPC programs are frequently responsible for reporting hospital-associated infections within their facility, it is the responsibility for reporting communicable diseases that is relevant to defense against biological attacks. Communicable disease reporting requirements (also known as Infectious Disease Reporting) vary within each state and even county, but overall, there are state laws requiring medical providers to report notifiable disease to local public health departments [1]. While there is variation in the lists of notifiable diseases that states require healthcare providers to report, there is a general consistency of common and concerning microorganisms that includes all Category A agents and 
a majority of those listed within the Center for Disease Control and Prevention's (CDC) bioterrorism agents page [2-5]. Within hospitals and healthcare facilities, it is generally the IPC program that is responsible for reporting communicable diseases to local public health authorities. While laboratory reporting can and does happen, it is mostly incomplete information and commonly limited to the positive laboratory result and the name of the patient. The legal responsibility of reporting the communicable diseases ultimately tends to fall upon the infection prevention and control program, which must not only report such diseases with complete patient and laboratory information, but also within the required time frame. State reporting laws dictate the list of notifiable diseases and their required reporting time frame. For example, in California, the California Code of Regulations requires a report for West Nile virus infection be submitted by electronic transmission, telephone, or mail within one working day of identification, while a human or animal case of plague requires immediate reporting by phone [6]. These lists mirror that of the CDC's National Notifiable Diseases Surveillance System (NNDSS). In essence, the reporting structure commonly begins with the IP performing surveillance based off laboratory reports and daily reports of admitting diagnoses for newly admitted patients. From this surveillance, reporting is performed to the local health department, which will then report to the state health department, which then reports it to the CDC. In short, national diseases surveillance and reporting frequently starts with hospital IPC programs.

IPC programs have a critical role in communicable disease reporting, which aids in public health response. The strength of the infection prevention and control program will be seen in their ability to perform more specific surveillance and real-time disease reporting. Poorly supported or minimally staffed IPC programs will likely rely solely on microbiology reports and might be delayed in their surveillance and reporting efforts. Some diseases, like Kawasaki syndrome, cannot be confirmed by laboratory tests, which can be challenging, but is overcome through daily chart review and a strong relationship with physicians and other clinical staff. Moreover, once a case is identified as a communicable disease that must be reported, the skilled IP will not only report the required information (e.g., laboratory results, demographics, contact info) but also relevant medical record information for the condition, which will include a timeline of illness, travel history, physician notes, etc. Such attention to detail facilitates a more rapid and efficient response from public health authorities. Overall, the infection prevention and control program within healthcare facilities is frequently the primary source of communicable disease reporting, which is a vital part of public health response during biological events.

\subsubsection{Coordination Among Internal Departments}

IPC programs are a connective tissue within the healthcare setting, working across professions and job roles to ensure the response to infectious disease threats is appropriate. It is the responsibility of the IPC program to ensure that clinical staff follow infection control guidelines, but also ancillary staff, which includes but is not 
limited to environmental services, facilities, sterile processing and disinfection, laboratory, security, or food services. IPC coordinates with these departments to ensure the proper education and compliance is ongoing as interaction with patients is not solely limited to healthcare providers. Aside from ensuring infection control practices are followed, the IPC program must also coordinate with different departments within the healthcare facility/system to ensure the proper expertise are being utilized. Consider the role of construction or facilities maintenance. A relatively simple task, like installing cabling or renovating a new space can bring with it substantial patient safety concerns due to dust, mold, etc. IPC must consider the full spectrum of affected people and departments during biological incidents, for example; during a public health emergency or treatment of a patient in enhanced precautions, IPC must coordinate with environmental services to ensure proper waste disposal is being performed. In the event of a pediatric patient with Ebola virus disease, IPC must ensure that Child Life Services are able to provide recommendations and guidance in regards to the unique needs of pediatric patients. Such unique situations require IPC response and coordination of expert opinions to ensure the most appropriate actions are taken. During an influx of patients with an infectious disease or in the wake of an attack, IPC will be an integral component to hospital response and coordination of efforts. In emergent situations, the Hospital Incident Command System (HICS) may be initiated, of which IPC plays a vital role in providing instructions and expert recommendations.

\subsection{Isolation Precautions and Personal Protective Equipment}

Isolation precautions are singularly one of the most critical components to preventing the spread of infectious diseases during patient care. The rapid isolation and adherence to personal protective equipment guidelines are imperative in daily patient care, but will become even more pertinent during infectious disease crises. Adherence to standard precautions and hand hygiene is fundamental for all patient interactions. However, additional transmission-based precautions may be needed during the medical care of patients with infectious diseases. Transmission-based precautions (isolation precautions) are often required in addition to standard precautions. Full information on microorganism-specific isolation precautions and detailed PPE directions can be found within several CDC and World Health Organization (WHO) publications. IPC recommendations follow CDC guidelines for isolation precautions and corresponding PPE based upon the identified microorganism (e.g., bacterial, viral, fungal). The recommendations within this chapter serve as broad guidance for utilization and compliance. Reference is made to the patient room, but isolation precautions should be applied to the area in which the patient is present (e.g., treatment room, post-procedural recovery bay). 


\subsubsection{Personal Protective Equipment}

Personal protective equipment (PPE) in the healthcare setting includes gloves, gowns, masks, shoe covers, and eye-protection. These items are the most common and frequently used PPE during patient care, but additional PPE may be recommended in emergent situations. PPE provides a mutual benefit to both patient and healthcare worker as it reduces the spread of diseases between the two parties. Correct utilization of PPE decreases the microbial burden in the environment as well, preventing the spread of disease through cross-contamination. PPE is mutually beneficial within the care process, which should be taught in training to encourage compliance.

Donning and doffing PPE is a critical component to efficacy. Improper use of PPE is common among healthcare workers, as the time to properly don can be taxing or because it is thought to be easier to continue wearing PPE if only leaving a patient's room momentarily (e.g., keeping a facemask on while running to the medication room). For PPE to be effective, it must be donned prior to entering the patient room and doffed prior to leaving or upon exiting (depending upon the layout of the care area) to avoid contamination of the hospital unit. PPE should always be changed between patients and should never leave the patient's room, regardless of how short a simple task may appear. If a patient is in isolation, anyone entering the room should don PPE, regardless of whether the planned task does not involve patient interaction or expected contact with the environment. Once in the patient's room, one cannot predict what will be needed or if an emergency will occur, meaning that PPE should be worn at all times while caring for a patient in isolation.

Isolation precautions and the required PPE range from standard precautions to airborne isolation, all of which have different and distinct requirements. Adherence to $\mathrm{CDC}$ isolation precaution guidelines and manufacturer recommendations for the PPE are imperative for the treatment of patients with infectious diseases. Regardless of origin, vigilant adherence to proper isolation and PPE guidelines will aid in infection prevention and control efforts, which can halt an outbreak in its tracks. PPE can and should be worn by visitors of the patient however, this is limited to PPE that does not require fit-testing (i.e., respiratory protection for patients with airborne microorganisms, like N-95 masks, should not be worn by visitors or patients as they require special fit-testing to ensure proper sizing). During hospitalization, the room of an contagious patient should have the proper signage on the door indicating which transmission-based precautions are being used so that all staff or visitors entering may wear the necessary PPE.

\subsubsection{Standard Precautions}

Standard precautions (also referred to Universal Precautions) are basic precautions that should be utilized on all patients as a means of reducing the transmission risk of, for instance, bloodborne pathogens [37]. The most basic premise is to use hand hygiene and assess the risk of a potential exposure to blood or other potentially 
contaminated material (e.g., urine, feces, breast milk) and use the corresponding PPE. Standard precautions encourage the healthcare worker to consider the tasks being performed and apply critical thinking to the risk of potential exposure and then select PPE based upon this assessment. For example, drawing blood typically only calls for gloves, but if the patient is thrashing or aggressive, the healthcare worker should consider eye protection. Another component to standard precautions is respiratory hygiene and cough etiquette. This lesson was developed and became essential during the SARS outbreak and has now become a staple within universal precautions. Respiratory hygiene and cough etiquette highlights the importance of education for healthcare workers, patients, and visitors, to ensure they cover their mouth and nose when coughing or sneezing, perform hand hygiene following exposure to respiratory secretions, and practice spatial separation of febrile persons with respiratory symptoms. The goal of standard precautions is to ensure that, at the most basic level, healthcare workers are protected against blood and other potentially contaminated material through hand hygiene and risk-based PPE utilization. Additional transmission-based precautions, such as contact isolation precautions, build upon standard precautions, meaning that standard precautions will always be used.

\subsubsection{Hand Hygiene}

The most basic and effective tool in the infection prevention arsenal is hand hygiene. Competent hand hygiene practices can prevent the transmission of diseases, making it the foundation for infection prevention [32]. Efforts to maintain compliance can be challenging as healthcare workers are expected to perform hand hygiene before and after patient interaction [30, 31]. The new WHO model for hand hygiene goes beyond the "before and after patient care" practice and emphasizes moments during the patient care process. The Five Moments of Hand Hygiene (frequently called "5 moments") model emphasizes a broader approach to hand hygiene that encompasses the intricacies of patient care. The WHO's 5 Moments model is increasingly becoming the standard practice and includes five moments in which hand hygiene should be performed: before patient contact, before aseptic tasks, after body fluid exposure risk, after patient contact, and after contact with patient surroundings [7]. Hand hygiene should also be performed after removing gloves. Gloves can easily be seen as a cure-all protective barrier however, hand hygiene should be performed following their removal as leakage during care and cross-contamination during removal is possible [8].

Hand hygiene is performed with either soap and water or alcohol-based hand sanitizer. According to the CDC, washing with soap and water for 15-20 seconds should be performed when hands are visibly soiled, after known exposures to Clostridium difficile, after known or suspected exposures to patients with diarrhea during a norovirus outbreak, when exposure to Bacillus anthracis is suspected or proven, before eating, and after using the restroom. If none of those situations are applicable, alcohol-based hand sanitizer is recommended and considered the most effective product. Alcohol-based hand sanitizer (composed of 60-95\% alcohol to 
maintain efficacy) is an easy and effective manner for hand hygiene that takes roughly 20 seconds to dry. Depending upon the situation, alcohol-based hand sanitizer can easily provide staff with an effective and timely method for cleaning their hands, which significantly improves compliance.

\subsubsection{Contact Precautions}

Contact isolation precautions are used for microorganisms that are transmissible through touch or contact with the patient or a contaminated item (fomite). Contact precautions include wearing a gown and gloves during interaction with the patient or their environment. The most common examples of infections in which contact isolation is used include diarrheal illnesses, methicillin-resistant Staphylococcus aureus (MRSA), and draining abscesses. Contact precautions should also be taken during the care for patients infected with known or suspected multi-drug resistant microorganisms, Human Parainfluenza virus (HPIV) infection in pediatric patients, poliovirus, vaccinia virus (adverse events following vaccination, like generalized vaccinia or conjunctivitis), etc. Broadly speaking, contact precautions, should be used for patients with diarrhea or draining wounds, and should be considered for children who have respiratory illnesses. The necessary PPE for contact precautions, gown and gloves, should be donned appropriately before entering the room, and removed at the threshold, prior to exiting. Depending on the make of the gown, it is important to follow manufacturers' recommendations for removal (like pulling away from the body to break any ties in the back.) and wear (i.e., wearing the gown over the shoulders, not hanging down around the elbows). Contact precautions are an effective practice for protecting the healthcare worker against infectious diseases that are spread through touch and fomites.

\subsubsection{Droplet Precautions}

Droplet isolation precautions are used to help stop the spread of microorganisms that are spread through tiny droplets that are disseminated through sneezing and coughing. The most common causes of droplet precautions are pneumonia, seasonal influenza, pertussis, and bacterial meningitis. Droplet precautions can frequently be confused with airborne precautions, but these precautions focus on those microorganisms that are transmitted via droplets during close proximity interactions [9]. To protect against droplet-spread microorganisms, a surgical mask should be worn while in the patient's room. The mask should be put on prior to entering the room and removed upon exiting the room. While most PPE should not be worn outside of the room, a surgical mask should be discarded in the trash after leaving the room [10]. Hand hygiene should be performed after the mask is removed upon exiting of the room. Masks should always be disposed of after leaving the room and should not be re-used for multiple patients as the mask is considered contamination after being touched. In emergent conditions, there have been situations that call for 
re-using masks if supplies are limited however, these should be evaluated during the event and not a common practice. The Association of Perioperative Registered Nurses (AORN), Food and Drug Administration (FDA), WHO, and CDC all recommend disposal of FDA-approved medical masks after single use and that healthcare workers should don a new mask for each contact with a new patient [11]. AORN highlights the hazards of reusing surgical masks by saving them or hanging them around the neck. Droplet precautions are an effective infection control strategy for preventing the spread of infectious diseases that are spread via droplets and close contact.

\subsubsection{Airborne Precautions}

Airborne isolation precautions are required during the care of patients who have or are suspected of having diseases like tuberculosis, measles, or chickenpox, which are caused by small organisms $(<5 \mu \mathrm{m}$ in diameter) that easily flow on air currents. Patients infected with such pathogens are capable of transmitting disease through respiratory secretions that are aerosolized during coughing, sneezing, suctioning procedures, etc. Airborne precautions are perhaps the most challenging as they require healthcare workers to wear appropriate respirators that have been fit-tested. Adherence to airborne isolation requires healthcare workers to be fit-tested to ensure the National Institute for Occupational Safety and Health (NIOSH)-approved N-95 or higher level respirators maintain a proper seal around the face. These masks should be donned prior to entering the room, removed after exiting the room, and then disposed of.

One of the most crucial aspects of airborne precautions is that the patient must be in an appropriate negative air pressure room. Negative pressure rooms (also called airborne isolation rooms or AIIR) are designated rooms with the proper engineering controls to ensure that aerosol particles containing microorganisms $<5 \mu \mathrm{m}$ in diameter are kept inside the room. It is vital to maintain negative air pressure, as it ensures that the air flow is from outside into the room, which prevents such microorganisms from being carried into the hallway and adjacent spaces (more information on negative air pressure rooms can be found in the section on environmental infection control). If a patient is suspected of having a disease that requires airborne isolation, it is critical to have them wear a surgical mask until a negative pressure room is available. One of the most common sources for exposures is delay in placing the patient into airborne isolation. Healthcare workers should don N-95 respirators while in the patient's room and the patient should wear a regular surgical mask during transportation or in an area that is not negative pressure. The use of a surgical mask for patients acts as a protection for those in the rest of the healthcare space.

Airborne isolation is perhaps the most prudent during bioweapons-related or high-consequence biological events. Patients with high-consequence pathogens, such as monkeypox, SARS, smallpox, viral hemorrhagic fevers, MERS, and influenza, require airborne isolation with some additional isolation precautions. Airborne 
isolation is the most challenging aspect of dealing with such biological threats as it requires the unique environmental controls that can pose challenging during an influx of patients with infectious diseases or in resource poor areas. A recent WHO report highlighted the role of healthcare facilities and infection control failures in MERS outbreaks, which underscores the consequences of PPE and infection control failures $[12,13]$. It is a common practice in healthcare that a patient with a cough/fever has to wear a mask until infectious etiologies can be ruled out. By simply having a potentially sick patient wear a surgical mask during the triage process, healthcare workers and other patients can be protected, regardless whether the patient has an infectious disease that is spread by droplet or airborne mechanisms. Although airborne isolation can be the most taxing in terms of ensuring properly fitted masks are used and negative pressure rooms are available, these are vital components to infection control for high-consequence and highly transmissible diseases.

\subsubsection{Combination or Enhanced Precautions}

In many cases, combining isolation precautions is most beneficial. As medical care grow is in complexity and more people are involved in patient care, there is a higher risk for spreading microorganisms via aerosol-generating procedures or contaminated surfaces/objects. Additional PPE, like boot/shoe covers, caps, etc., may be utilized in such isolation practices. However, proper PPE donning and doffing practices should always be followed [33]. Some microorganisms, like Human herpesvirus 3 (HHV-3, varicella-zoster virus), require a combination of airborne and contact isolation due to the nature of the illness they cause and transmission mechanisms. It is also common in pediatric care to combine contact and droplet isolation during the care of patients with respiratory viruses (e.g., human metapneumonvirus, respiratory syncytial virus, rhinovirus) as children frequently spread such microorganisms through touch. For example, according to CDC guidelines, respiratory infectious diseases (except for influenza, pertussis, etc.) only require standard precautions in adults. However, in infants and young children, standard plus contact isolation is encouraged, which emphasizes the need to assess the patient population when considering isolation precautions during care. Combination precautions are especially beneficial if microorganisms are isolated from multiple sources, such as a patient with Carbapenem-resistant Enterobacteriaceae isolated from both a draining wound and an endotracheal secretion. Typically, contact precautions are sufficient. However, if the microorganism is isolated in respiratory secretions, it is suggested that droplet precautions also be used.

There is an increasing practice of combining and enhancing isolation precautions for severe, high-consequence, or novel infections as research is ongoing regarding their transmission mechanisms. Enhanced isolation goes beyond just combining transmission based precautions, but adding additional measures and processes [33, 34]. Ebola virus disease, SARS, MERS, and many other diseases require a combination of isolation precautions that include airborne and contact prevention measures, but also 
enhanced isolation practices that include additional PPE and precautions. Novel or emerging infectious diseases may require an amalgamation of isolation precautions, especially in advanced medical settings. The 2013-2016 Ebola virus disease outbreak highlighted the challenges of managing patients in American hospitals and required substantial revisions to existing PPE and isolation guidelines. At the most rudimentary level, isolation precautions should be designed around the transmission mechanisms of the infectious disease. SARS and MERS coronaviruses are primarily spread through respiratory droplets, which caused significant issues in the early stages of outbreaks due to delayed airborne isolation. On the other hand, Ebola virus disease cases within the United States revealed gaps in isolation guidance and preparedness when responding to patients with emerging infectious diseases. Although aerosol-generating procedures were a topic of concern for SARS and MERS, respiratory precautions were already considered for isolation precautions. The limited experience and knowledge among healthcare workers surrounding Ebola virus disease also posed unique challenges for training on enhanced isolation precautions and PPE during the 2013-2016 outbreak. Overall, enhancing isolation precautions is a helpful strategy for handling a novel or emerging infectious disease, especially if newer or more invasive medical procedures or pediatric patients are involved. One of the most common lessons infection preventionists highlight for clinical staff is that it is always better to quickly initiate isolation precautions and remove them later than find out hours or days into a patient's care that they were contagious. Patients may voice frustration with being placed in isolation, but delay in isolating a patient who potentially has an infectious disease, runs the risk of furthering disease transmission.

\subsubsection{Isolation and PPE Compliance in Visitors}

Visitors play a significant role in the well-being of hospitalized patients however, they also can facilitate the transmission of infectious diseases. Healthcare facilities will ultimately need to grapple with balancing patient satisfaction and the potential for visitors to act as amplifiers of disease, especially during outbreaks. Adherence to isolation precautions and hand hygiene is vital among healthcare workers, but the role of visitors has also become a topic of concern. There is substantial evidence for the horizontal transmission of even multi-drug resistant microorganisms involving the hands and attire of healthcare workers. However, consideration must also be given regarding the role of visitors in infection prevention and control [14]. While there is no universal recommendation for handling visitor compliance to standard or more advanced precautions, it is encouraged that those visiting a contagious patient should use PPE for their own safety. The Society for Healthcare Epidemiology of America (SHEA) has provided recommendations that highlight the challenges in enforcing visitor compliance, but also notes that it is crucial to consider the infectious microorganism when dealing with visitors who are unwilling to follow precautions. Proper education and guidance on PPE donning and doffing should be given to visitors. However, hand hygiene is perhaps the most important and likely practice to be followed. SHEA notes that "hospital visitors may play a significant 
role in the ecology of healthcare-associated infections" but few visit more than one patient, which makes the transmission between visitors and multiple patients less likely [14]. There is a consistent challenge in enforcing compliance with visitors and little research that has focused solely on their compliance and role within healthcareassociated disease transmission. Several things should be considered to address visitors of patients in isolation for an infection. If the visitor is a household contact and likely to already be exposed the concern then becomes if they are already infected and potentially contagious. If the visitor is a breast-feeding mother who is involved in the care of the patient, it is unlikely isolation precautions can be enforced. Many hospitals within the U.S. also make it a practice to screen visitors during respiratory virus season to prevent symptomatic visitors from exposing patients or staff [35]. This practice should be considered for visitors of such patients, especially if they are house-hold or close contacts and may be a source for further transmission.

Patients with high-consequence diseases, like Ebola virus disease or SARS, may pose a unique challenge for healthcare workers in terms of visitors and isolation precautions and should addressed on a case by case basis. CDC recommendations for visitors of Ebola virus disease patients include that healthcare workers should "avoid entry of visitors into the patient's room" and that visits should be scheduled and controlled [15]. Visitors played a significant role in furthering the spread of SARS and MERS during outbreaks and consideration should be given to restricting the number of visitors during such events. A recent WHO report on MERS found that of the cases between December 2016 and July 2017, 31\% were associated with transmission in a healthcare facility, including healthcare workers, patients sharing rooms/wards with MERS patients, and visitors [12, 13]. CDC guidance for infection control during treatment of patients with SARS also encourages limiting visits to those who are necessary for the patient's emotional well being. Ensuring public health during outbreaks inherently trumps visitation [16]. Visitors should be educated and encouraged to wear the necessary PPE when visiting an isolated patient. At the bare minimum, it is prudent to educate and encourage visitors to practice hand hygiene upon entering and exiting the patient's room.

Airborne isolations pose a challenge for visitor compliance. Since hospitals can not support fit-testing visitors for N-95 masks or other respirators, each situation should be evaluated on an individual basis. Surgical masks can provide some protection but will not provide a true seal. It is a common practice for many healthcare institutions to allow house-hold contacts to visit a patient in airborne isolation without a mask (or wearing a surgical mask) since exposure has likely already occurred. In this situation, consideration should be given for exposure from that visitor to healthcare workers and others after they have left the negative pressure room. It may be prudent to have visitors in such situations wear a surgical mask upon exiting the patient's room and wear it until they exit the hospital.

Visitor adherence to isolation precautions is challenging for even the most common hospitalizations. Staff compliance and vigilance can be a strong rolemodel and reminder for visitors, especially in regards to hand hygiene. In the event of a bioweapons-related attack or outbreak of a highly infectious disease 
(i.e., Ebola virus disease), visitor protocols should be established. Unique events and microorganisms can pose a challenge for isolation precaution compliance and recommendations may need to be modified for novel or specific situations (i.e., caring for a pediatric patient and maintaining parent/guardian visitor isolations). Changes to IPC guidelines for such unique situations should be done in conjunction with CDC (and other expert) guidance and discussed in a multidisciplinary setting to ensure support is facility-wide. In these situations, allowance of visitors and PPE recommendations should be made on a case by case basis. Visitors do play a significant role in infection prevention and control within the healthcare environment, which makes their adherence to standard precautions and isolation precautions important and challenging.

\subsubsection{Discontinuation of Isolation Precautions}

Transmission-based precautions should be maintained until the patient is no longer considered capable of transmitting the disease. The CDC's Guidelines for Isolation Precautions is an excellent resource for duration of isolation. Consideration should be given for immune status of the patient as immunocompromised patients may need to be isolated for longer periods of time due to lengthier microorganism shedding.

\subsection{Patient Placement and Cohorting}

Patient placement is a vital component to infection control measures, especially during an influx of ill patients. Isolation practices and cohorting (grouping like patients in a shared hospital room or designated unit) plays a significant role in reducing the microbial burden within the healthcare environment. Patient cohorting is a common practice during outbreaks or limited availability of negative pressure rooms however, it is important to consider transmission-based precautions during the assignment of patient rooms and establishing cohorted units.

\subsubsection{Placement of Patients}

Isolating patients with the appropriate transmission-based precautions is often done during admission. However, if patients are transferred to the healthcare facility and there is already suspicion of an infectious disease, this may also determine where the patient is placed. There is a myriad of components that impact patient placement and such efforts should be performed with IPC in mind.

Although many healthcare facilities have private rooms, there is always the possibility that patients may have to share rooms. Efforts should be made to place patients with infectious diseases that are transmitted via the contact, droplet, or airborne route, in private rooms. Consideration for route of transmission should always be made during patient admission, which would determine both isolation 
status and patient placement. Patients needing airborne isolation require rooms that maintain negative pressure, which are typically only a handful of rooms per care area. These patients should be place within private negative pressure rooms. There is an increasingly common practice of requiring private rooms for patients infected with highly resistant microorganisms, such as carbapenem-resistant Enterobacteriaceae (CRE) or multidrug-resistant TB (MDR-TB). Patient placement should be determined with transmission-based precautions in mind and efforts should be made to ensure patients with highly contagious or high consequence pathogens (e.g., Ebola virus, SARS and MERS coronaviruses) are in private isolation rooms per CDC guidelines.

\subsubsection{Cohorting of Patients and Staff Assignments}

Despite our best efforts, during peak respiratory virus season or an influx of patients, private room availability may be constrained. Cohorting is a common practice during times in which room availability may be scarce. There are two kinds of cohorting; sharing of private rooms or grouping patients within a defined entire hospital unit. If private rooms are not available, patients infected or colonized with the same microorganism (e.g., influenza A virus, MRSA) can be cohorted together to share a room. During respiratory virus season, cohorting based off clinical symptoms is often utilized. While it was necessary to cohort Ebola virus disease patients during the Western African 2013-2016 Ebola virus disease outbreak, CDC guidelines for U.S. hospitals is that patients should be placed in a private room. If there should be an influx of patients with a high consequence pathogen, consideration for cohorting within the same room should be made on a case-by-case basis with infection prevention and control coordinating with the appropriate public health authorities.

During an outbreak or influx of patients in which hospital bed capacity is strained, cohorting can be utilized for an entire hospital unit or well-defined space. Designated hospital units can be cohorted for infected patients. These units should either be dedicated to patients affected by an outbreak (and the same microorganism) or of all patients in isolation who are cohorted within their rooms based off the previously mentioned criteria. This is an effective practice for segregating the patients and their visitors from healthy/non-infected patients. Cohorting is also an effective strategy for reducing the risk of cross-contamination by staff. The CDC notes that "cohorting healthcare personnel to care only for patients infected or colonized with a single target pathogen limits further transmission to uninfected patients but is difficult to achieve in the face of staff shortages in hospitals and residential healthcare sites. However, if continued transmission is occurring after implementing routine infection control measures and creating patient cohorts, cohorting of healthcare personnel may be beneficial." [17]. Designated staff can be assigned to cohorted patients or units. It is highly encouraged that healthcare workers (especially nurses) caring for cohorted patients, should not also be assigned to patients who are immunocompromised. Cohorting in single rooms or in well-defined units can be an effective mechanism for reducing the spread of infectious diseases during patient care. 


\subsubsection{Designated Staff For Specific Microorganisms}

The Ebola virus disease outbreak in 2013-2016 highlighted the role of healthcare worker transmission, not only between themselves, but also among patients. CDC guidelines encouraged healthcare facilities to minimize staff with direct patient care. It was encouraged that those staff caring for a person under investigation (PUI) or infected with Ebola virus, be dedicated to that patient as to minimize the potential for additional exposure. During the 2011 outbreak of carbapenem-resistant Klebsiella pneumonia at the National Institute of Health's Clinical Center, IPC staff adopted a series of control measures that included cohorting, limiting activities of hospital staff exposed to infected patients, and hiring monitors to ensure healthcare providers were wearing appropriate PPE and using hand hygiene [18]. Facilities should have policies developed and in place for those staff who may be exposed during their duties. Incidents involving bioweapons, novel or emerging infectious diseases, or high consequence pathogens, will surely require unique guidelines that could test the capacity for hospitals. It behooves healthcare facilities and preparedness efforts to consider staffing and cohorting needs during such challenging situations.

\subsection{Patient Screening and Movement}

The flow of patients from triage to discharge also poses vulnerabilities for infectious disease transmission. The initial screening of patients can be an effective infection control measure for rapid isolation and PPE utilization. Proper communication and triage questions can aid in the rapid identification of patients with increased risk for infectious diseases. Hasty identification of a patient with a potential infection within their first moments within the healthcare facility can substantially decrease the risk for additional exposure. Once admitted to a healthcare facility, patients are frequently transported for diagnostic tests and care. The transportation of a patient can also act as a facilitator for disease transmission if infection control practices are not maintained.

\subsubsection{Emergency Department Screening and Triage}

The initial interaction a patient within a healthcare facility or urgent care unit is the first opportunity to identify risk for infection. The triage process determines the degree of urgency of the patient's condition, which then decides the order they are seen. During this process, there are many opportunities to not only identify patients with potentially contagions infections, but also rapidly isolate them to prevent further exposure to staff and other patients. It is encouraged for triage staff to provide patients presenting with a cough, (i.e., a potential respiratory infection) a mask to wear during their time in a waiting room. This simple act can be hugely preventative, whether the patient has influenza, MERS, or TB.

While the triage process primarily focuses on medical questions, this is also an opportunity for questions regarding international travel and fever. Since the SARS 
outbreak in 2002, it has become a relatively common practice to incorporate a question within the patient screening/triage process regarding international travel and fever. Travel history is always an important component to the patient's medical history but we should not forget its significance during triage and patient screening. Presence of fever is a standard part of the medical triage and intake during initial screening and if the patient is febrile, questions regarding travel history become that much more important. There are a mixture of recommendations regarding processes, but it is consistently thought that if a patient with an acute illness (e.g., fever within 2 weeks, diarrhea) has traveled outside the U.S. within the last 21 days, the patient should be isolated. Isolation should be based upon the presenting signs and symptoms but providers should also consider the area of travel and if there are ongoing epidemiologically-significant outbreaks. During outbreaks, like those of SARS and MERS, screening and triage can include questions regarding not just travel, but also exposure or close contact with a sick person who recently traveled to those areas or was infected. The screening and triage process is especially beneficial for early detection and isolation of potentially infectious patients because it can easily be modified. Screening questions can be added or removed based upon healthcare needs. While the standard questions remain the same, questions about specific travel or exposures can be added during times of public health crises. Questions related to travel history should be maintained during all times though and it is encouraged that patients with an acute illness and international travel within 21 days, be placed in a private room until further evaluation by a healthcare provider. Measles and TB are frequent suspected in patients with respiratory symptoms and a recent international travel history. However, the inclusion of travel history in medical triage and screening can also potentially capture cases of avian influenza, MERS, viral hemorrhagic fever, etc.

\subsubsection{Transportation of Patient Within Hospital}

Patients are frequently transported throughout the hospital during their care, which can make maintaining isolation precautions challenging. It is encouraged for patient transportation to be limited, but there are several recommendations to further reduce the spread of disease during patient movement. Using appropriate barriers is crucial during the transportation of an infectious patient.

Patients in contact isolation are encouraged to change into a new hospital gown or attire and should be transported on a wheelchair or bed covered with a clean sheet (or other barrier). Infectious skin lesions should be covered. The transporter should wear the appropriate PPE while assisting the patient into or out of the covered wheelchair or bed, which should then be removed upon exiting the room (gown and gloves are not required during the transport of patients in contact precautions). The CDC notes that "when transport or movement in any healthcare setting is necessary, ensure that infected or colonized areas of the patient's body are contained and covered." [17]. Those patients in droplet or airborne isolation should wear a mask during the transportation process. It is important that notification of the 
patient's isolation status be done to the receiving department or healthcare personnel to facilitate the continuation of isolation precautions.

\subsubsection{Exposure Response}

Despite best efforts, patients are not always isolated or transported appropriately. If a patient with an infectious disease has potentially exposed a considerable number of staff and other patients, infection prevention and control programs may be used to the contain further spread. All exposures are not equal, meaning that MRSA is a common microorganism within the community and an exposure response is often not warranted, whereas exposure to mycobacteria or a more severe illness may require follow-up. These responses should be determined by the IPC program, Occupational Health, and the medical directors associated with these programs in conjunction with hospital administration. Post-exposure prophylaxis may be necessary for staff and patients, but medication distribution should be determined by the appropriate hospital experts. Hospitals and IPC programs should have a functioning process for determining patients with exposure to specific staff or in other patients (i.e., those in the waiting lobby of an emergency department). Commonly, exposure monitoring and notifications are used for patients, visitors, and staff exposed to measles, pertussis, etc., as post-exposure prophylaxis is recommended. IPC programs might monitor admitted patients who were exposed or potentially isolate them during the incubation period. Exposures may also be the result of infectious healthcare staff, which may require patient notification. Overall, these events should be addressed on an individual basis and adequate response measures should be applied to reduce the time between exposure and follow-up.

\subsection{Environmental Infection Control}

Although isolation and PPE utilization are a significant portion of preventing the spread of disease during hospitalization or medical treatment, the role of environmental transmission should not be forgotten. Many diseases can be spread through fomites and poor consideration for the environmental aspects of infection control only fuel transmission. There is considerable literature on the recommendations and guidelines for environmental infection control, specifically by the CDC, OSHA, WHO, and APIC. This section will provide a very high-level overview of these publications. The goal of this section is to raise awareness for environmental mechanisms of transmission and encourage consideration during the development of preparedness and response plans. Healthcare environmental components, such as building design, waste management, disinfection and sterilization, and air monitoring, are critical to reducing the spread of microorganisms, but the ability to make changes may be hindered by high costs and other restraints. 


\subsubsection{Cleaning, Disinfection, and Sterilization}

Cleaning and disinfection of non-critical surfaces is a basic premise of infection control practices, especially standard precautions [17]. These practices become even more significant when caring for patients with infectious diseases. Cleaning is the process of removing pathogens, whereas disinfecting kills germs on the surface, and sanitizing lowers the number of pathogens on an object to a safe level. Rutala and Weber provide an extensive outline of such methods for cleaning, which include:

- Sterilization: destroys all microorganisms, including bacterial spores

- High-level disinfection: destroys all microorganisms except high numbers of bacterial spores

- Intermediate level disinfection: destroys vegetative bacterial, mycobacteria, most viruses, most fungi, but not bacterial spores

- Low-level disinfection: destroys vegetative bacteria, some fungi and viruses, but not mycobacteria or spores.

Such practices should be closely followed, especially in the event of novel or severe illnesses requiring enhanced precautions. Disinfection recommendations may be revised based upon recommendations from OSHA and the CDC, as was seen during treatment of patients with Ebola virus disease in U.S. hospitals. Environmental contamination can be a significant mechanism for disease transmission during patient care and invasive procedures can increase this substantially. Poorly disinfected or sterilized equipment has been the culprit of many healthcareassociated infections and outbreaks. High-touch surfaces or objects, like sinks and bedrails, require frequent cleaning and disinfection. Frequency and intensity of cleaning and disinfection may also need to be increased depending up on the "degree of environmental contamination" [17]. Environmental Protection Agency (EPA)registered disinfectant should be used to combat the environmental contamination that occurs during patient care. It is important to note that certain pathogens may be resistant to routine disinfectants (e.g., rotaviruses, noroviruses, Clostridium difficile, Bacillus anthracis), meaning that healthcare workers should ensure the cleaning product they are using is effective against the specific microorganism. Disinfection and sterilization of medical equipment should always follow manufacturer recommendations and be handled with care.

There are frequently forgotten objects and surfaces that are common culprits for contamination and should be highlighted during routine cleaning. Computer keyboards, stethoscopes, and clinical work stations are all areas that routinely have a high microbial burden and are frequently neglected during cleaning. There is extensive literature on practices for disinfection and sterilization, but at the most basic level, preparedness and response efforts should work to ensure routine cleaning and disinfection is done appropriately and correctly [36]. In emergency situations, a quick lesson is always to work "from clean to dirty" and consider all surfaces and objects. Environmental contamination is a continued source for disease transmission in healthcare. These practices should be strengthened during routine 
care and also refined to meet special needs during public health emergencies and care of patients in enhanced precautions.

\subsubsection{Ventilation Systems}

Hospital ventilation systems are a critical component to patient safety. These systems maintain negative pressure within airborne isolation rooms and positive pressure in operating/procedure rooms, as well as protective precaution rooms (for severely immunocompromised patients). Negative pressure rooms will become a critical need in outbreaks of microorganisms that require airborne isolation. These rooms should have high-efficiency particulate air (HEPA) and should also follow CDC guidelines for environmental infection control regarding air exchanges per hour (ACH) (i.e. $\geq 12 \mathrm{ACH}$ for new construction as of 2001 ; $\geq 6 \mathrm{ACH}$ for construction before 2001) and be monitored in accordance with engineers' and manufacturers' recommendations [19]. The negative pressure rooms should maintain air pressure by being properly sealed and are encouraged to have self-sealing doors and ante-rooms. The heating, ventilation, air conditioning (HVAC) systems should also have humidity monitors to ensure the moisture levels are adequate as to discourage microbial growth. The CDC has specific recommendations for air-handling systems in healthcare facilities, which are vital to patient safety and reduction of disease transmission. In the event of a biological attack or outbreak, ensuring that these systems are operational and can maintain proper negative pressure in designated rooms will be of the utmost importance. Although negative pressure rooms are limited on each hospital unit, there is potential to convert entire units to negative pressure through adjustments to the ventilation system and sealing of the unit [20].

\subsubsection{Environmental Services}

Environmental cleaning, waste management, and handling of textiles are all components of environmental service work in healthcare. These duties are especially important upon discharge of the patient when a deep clean of the room is required (commonly called "terminal cleaning"). Within a single day of admission, the composition of a hospital room's microbes begin to change and mirror that of the patient [21]. Combatting the "bacterial communities" that develop within the healthcare environment is an ongoing struggle that emphasizes the important of adequate and consistent environment services. Environmental services staff frequently manage these tasks and should be aware of the patient's isolation status so that staff utilizes proper PPE and ensures use of proper Environmental Protection Agency (EPA)-registered disinfectants (example: Clostridium difficile requires the use of bleach products to kill spores). Typically, hospitals have contracts with linen services and waste management companies (for both regular and medical waste). However, practices may change during the care of patients with high consequence 
pathogens that require enhanced isolation. CDC guidelines regarding medical waste, dishes and eating utensils and textiles all note that these objects have not been implicated in SARS coronavirus transmission and that routine procedures should be utilized [22]. In the case of Ebola virus, recommendations were significantly different. For example, despite Ebola virus forming enveloped virions (which are susceptible to a wide range of hospital disinfectants), no EPA-registered disinfectants had labels that specifically cited efficacy against the virus. Consequently, CDC recommendations were changed to encourage use of EPA-registered disinfectants that were effective against viruses that form non-enveloped virions, which are more resistant to hospital germicidal disinfectant cleaning products. CDC recommendations also included discarding of all textiles to avoid staff exposure during the laundering process [15]. Many hospitals developed Ebola virus preparedness plans that placed environmental service duties more on the clinical staff caring for a PUI instead of potential exposing additional staff. Environmental services practices should be closely followed during routine patient care but may require modification during the care of patients in enhanced precautions or during outbreaks.

In the pursuit of more effective and efficient cleaning and disinfecting processes, newer technologies have been utilized, like that of ultraviolet disinfection technology and copper-infused linen. Environmental monitoring to check room cleaning quality is also a frequently used measure to test true cleaning of the space. Overall, environmental infection control by means of environmental services staff, textile care, and waste management are critical components to breaking the chain of infection. The efficacy of these duties and the adherence to transmission-based precautions when working throughout a healthcare facility is a monumental task and becomes even more critical during outbreaks.

\subsubsection{Water}

There is a considerable amount of literature and regulatory guidance on environmental infection control as it relates to water however, this section will focus on only a handful. Firstly, facilities should consider the mechanisms for preventing the spread of waterborne microorganisms through proper hand hygiene, cleaning of sinks, avoidance of decorative fountains and fish tanks in patient-care areas, and maintaining proper temperatures for hot and cold water [19]. Should water disruptions occur, corrective decontamination of the hot water system may be necessary, which can include flushing the water lines and outlets with chlorine and/or superheated water. Hospitals should have contingency plans for water in the event that water disruptions are prolonged due to intrusions or contamination.

\subsubsection{Construction and Renovation}

Construction, renovation, remediation, repair, and demolition within a healthcare environment can be sources for infection. All work must be reviewed by infection 
prevention and control to coordinate prevention methods that include containment barriers and post-construction cleaning. Such efforts are necessary as demolition can lead to airborne infections associated with fungal spores in immunocompromised patients. Moreover, if the proper planning is not done, engineering outages can impact negative pressure rooms, which decreases their efficacy. Conversion of spaces to negative pressure wards or specialty isolation units should be planned and tested ahead of time to ensure proper ventilation is possible.

\subsection{Challenges}

\subsubsection{Poor Utilization and Support}

In the day-to-day world of medical care, infection prevention and control practices, like hand hygiene, frequently have poor compliance [23]. While there are a multitude of issues that IPC programs face, perhaps the most damning one is internally. Typically, IPC programs are seen as cost centers and not revenue generators, which frequently translates to lackluster support and prioritization [24]. Medicine and healthcare are competitive markets, meaning that those programs seen from this light are likely to receive cuts. While there have been studies evaluating the cost of healthcare-associated infections in relation to that of an IPC program, there is a considerable gap within the research regarding IPC programs and response during outbreaks or cases of high-consequence pathogens. Given that a single healthcareassociated infection (HAI), like a surgical-site infection, can cost upwards of $\$ 50,000$ and preventing just a handful of these infections can pay for the entire budget of an IPC program, it is not unreasonable to think that such cost-saving could be applied to biothreat response within hospitals [25]. The cases of Ebola virus disease in Dallas, Texas, in 2014 exposed the lack of preparedness within U.S. hospitals, poor compliance with infection control, and the cost of treating such a severe illness with enhanced precautions. It was estimated that the cost of treating a hospitalized patient with Ebola virus disease in the United States is $\$ 30,000$ per day [26]. Despite spending $\$ 60$ billion on biodefense since 2001 to prepare for biothreats, a single imported case of Ebola virus disease revealed deep breaks within U.S. preparedness efforts [27]. During the 2013-2016 Ebola virus disease outbreak, it frequently fell on the IPC programs to coordinate hospital-wide preparedness and response efforts to ensure facilities were prepared to identify, isolate, and potentially treat a patient. While such events highlight the importance of IPC programs and the diversity of their responsibilities, for many in healthcare the looming threat of Ebola has passed and so has the support. The focus for many IPC programs has gone back to preventing HAIs and Ebola virus disease response plans have been neglected. A 2015 APIC survey found that $92 \%$ of IPC program leaders believe they were more prepared than the previously year to receive a patient with a highly infectious disease [28]. While this is encouraging, would hospitals and IPC programs feel the same today, several years after the 2013-2016 Ebola virus disease outbreak? There is a substantial need within healthcare to truly support IPC 
programs for not only day-to-day HAI prevention, but also as a means of establishing an all-hazards approach. Infection prevention and control, as shown within this chapter, involves collaboration with many internal and external partners to ensure patient safety. IPC programs are sentinels within hospitals, alerting internal and external partners of issues, so that they may form a collective response effort. It behooves bio-preparedness efforts to utilize IPC programs and consider IPC practices as a vital resource for disease control, medical countermeasures, and surveillance.

\subsubsection{Invasive Medical Interventions}

While advances in medicine have given us the capabilities to increasingly counter disease, the invasiveness of certain medical procedures and treatments can pose substantial infection control risks. The treatment of Thomas Duncan, the first Ebola virus-diagnosed patient in the United States, in a seemingly "regular" hospital without specialized training or facilities to handle a patient under enhanced precautions, points to this very dilemma. In the weeks following his hospitalization, there were conversations in hospital around the U.S. as to the safety and ethical considerations for more invasive and riskier medical interventions during the treatment of a patient with such an infectious disease. Medical interventions like invasive surgery, dialysis, insertion of central lines, ventilation, extracorporeal membrane oxygenation (ECMO), or even labor and delivery, all carry inherent risks for the staff performing them. The outbreaks of SARS and MERS coronaviruses, and Ebola virus disease all challenged PPE and isolation recommendations, requiring changes to protect healthcare workers and visitors during treatment, especially those interventions that generate aerosols. These outbreaks have taught vital lessons regarding the life-saving capabilities of modern medicine and technology, but also reveal a lack of preparedness consideration for medical interventions in patients with such severe and uncommon diseases. Future efforts should establish treatment and procedure plans with these diseases in mind, drawing on lessons learned and creating new protocols so that afflicted patients may receive the full range of medical care and healthcare workers can perform such work with minimal risk.

\subsubsection{Conflicting Guidelines}

Perhaps one of the most challenging aspects of infection prevention during the Ebola virus disease response in American hospitals was the continuous evolution of guidelines for isolation, PPE, disinfection, handling of medical waste, etc. The Blue Ribbon Study Panel on Biodefense highlighted this very issue in its recommendations, pointing to the "need for a standardized process to develop and issue clinical infection control guidance for biological events." [29]. The recommendations from the CDC regarding isolation precautions, specifically the use of negative pressure rooms/airborne isolation and the necessary PPE, were an evolving 
process in the days following Thomas Duncan's hospitalization. As an infection preventionist, these days were taxing due to the constant scramble to not only train staff on the appropriate donning and doffing procedures, but even acquire the correct PPE. Novel diseases like SARS and MERS also tax the public health and healthcare response systems in terms of providing adequate recommendations for isolation. While these fluctuations are understandable, in the event of a biological attack, fear and hysteria will likely play a larger role than in naturally occurring outbreaks, which makes the stability of IPC recommendations that much more important. Healthcare workers must feel confident in not only their training, but also the efficacy of their PPE. It is crucial that future efforts include stronger processes to standardize infection control recommendations and guidelines during these critical times.

\subsection{Infection Control in Biodefense Efforts: Minimal Focus Despite Maximum Return}

Biodefense and global health security efforts work to strengthen capacity to respond to infectious disease threats. These efforts are imperative to preventing, detecting, and responding to biological events, whether they are intentional, accidental, or natural. Within these plans, there is often a considerable gap regarding the role of infection prevention and control and the programs within healthcare facilities. There have been several reports and recommendations in recent years to strengthen biodefense efforts. The Health Sector Resilience Checklist for High-Consequence Infectious Diseases from the Johns Hopkins Center for Biosecurity points to the importance of infection control practices and their significance, but there is little overall mention of the programs within hospitals. This checklist focuses on the adherence to infection control practices, like that of the Blue Ribbon Study Panel on Biodefense report, but tends to neglect the role of these programs. The same can be said of more recent pandemic influenza and hospital preparedness plans. Unfortunately, there tends to be a gap in the literature regarding IPC within biodefense efforts and although most focus on larger components (disease surveillance, PPE, medical countermeasures), the utilization of IPC programs should not go ignored. Hospitals already have this tool available and it behooves preparedness efforts to employ it.

There is a continuous trend of pointing to infection prevention and control processes and neglecting the programs responsible for ensuring they are followed. The systemic gap in preparedness efforts citing infection prevention and control practices but failing to account for the program and role of infection preventionists reveals a fundamental lack of truly understanding the role of these programs and internal hospital operations. Biodefense efforts inherently include infection control practices as they are the foundation for preventing the spread of disease, but this gap regarding the inclusion of IPC programs does them a disservice. Hospitals already struggle with following IPC practices to reduce the spread of infection. Biodefense efforts should strengthen the existing IPC infrastructure and ensure processes are effectively followed. If hospitals are already struggling with hand hygiene and PPE compliance, how will they manage bioterrorism acts or pandemics? 
The problem is twofold: current compliance to infection control processes is often substandard, and the IPC programs are frequently under-utilized and/or -prioritized. Biodefense efforts should seek to increase infection prevention and control efforts and strengthen processes while building upon the existing IPC program infrastructure that can be an effective tool. Infection prevention and control is a significant resource in the biodefense arsenal and should be seen as an independent component, like surveillance and medical countermeasures. These programs are already in place and while they focus on the day-to-day problems of HAIs, they can be utilized to better serve and strengthen the U.S. healthcare infrastructure and thus preparedness. Strengthening IPC programs and processes benefits health security whether it is a local outbreak, an act of bioterrorism, or a pandemic.

Hospitals are the frontline for biological events and their capacity to adequately detect and respond to such threats can either halt or help the spread of disease. Infection prevention and control practices are existing guidelines and recommendations that seek to combat disease transmission during medical care. IPC programs within hospitals work to ensure staff are educated on and compliant with such practices to protect patients, visitors, and healthcare workers. These programs are also an integral source for infectious disease surveillance and a connective tissue within hospitals, making them a significant contributor to public health surveillance. Outbreaks of SARS, MERS, Ebola virus disease, and multi-drug resistant microorganisms, have all revealed the importance of IPC and the devastating consequences of poor compliance. Continued failure to prioritize IPC programs and practices, as well as the role they serve within outbreaks and growing biological threats, will only serve to weaken preparedness and response efforts. Defense against biological threats requires strong infection control practices, programs, and the ability to adequately employ these efforts prior to and during public health emergencies. Healthcare is a critical infrastructure, meaning that if hospitals are amplifiers for disease transmission and sources for negative patient outcomes, the impact can be devastating. Infection prevention and control is an essential resource against all biological threats, regardless of their origin.

\section{References}

1. Doyle T, Glynn K, Groseclose S. Completeness of notifiable infectious disease reporting in the United States: an analytical literature review. Am J Epidemiol. 2002;155(9):866-74.

2. Centers for Disease Control and Prevention. Bioterrorism agents/diseases. 2017. https://emer gency.cdc.gov/agent/agentlist.asp. Accessed 7 Apr 2017.

3. Centers for Disease Control and Prevention. Clean hands count for healthcare providers. 2017. https://www.cdc.gov/handhygiene/providers/index.html. Accessed 19 May 2017.

4. Centers for Disease Control and Prevention. Standard precautions for all patient care. 2017. https://www.cdc.gov/infectioncontrol/basics/standard-precautions.html. Accessed 19 May 2017.

5. Centers for Disease Control and Prevention. Handwashing: clean hands save lives. 2017. https://www.cdc.gov/handwashing/show-me-the-science-hand-sanitizer.html. Accessed 16 June 2018. 
6. California Department of Public Health. Reportable diseases and conditions. 2017. https:// archive.cdph.ca.gov/HealthInfo/Pages/ReportableDiseases.aspx. Accessed 10 July 2017.

7. World Health Organization. Five moments for hand hygiene. 2006. http://www.who.int/gpse/ tools/Five_moments/en/. Accessed 19 May 2017.

8. Korniewicz D, Kirwin M, Cresci K, Larson E. Leakage of late and vinyl exam gloves in high and low clinical settings. Am Ind Hyg Assoc J. 1993;54(1):22-6.

9. Salath M, et al. A high-resolution human contact network for infectious disease transmission. Proc Natl Acad Sci U S A. 2010;107(51):22020-5.

10. Association for Professionals in Infection Control and Epidemiology - APIC. Rules for standard and isolation precautions. 2017. http://professionals.site.apic.org/10-ways-to-protectpatients/follow-the-rules-for-isolation-precautions/. Accessed 3 April 2017.

11. Committee on the Development of Reusable Facemasks During an Influenza Pandemic, the National Academies. Reusability of facemasks during an influenza pandemic. Washington, DC: The National Academies Press; 2006.

12. World Health Organization. Evidence of hand hygiene to reduce transmission and infections by multi-drug resistant microorganisms in healthcare settings. http://www.who.int/gpsc/tools/ Five_moments/en/. Accessed 19 May 2017.

13. World Health Organization. WHO MERS-CoV global summary and assessment of risk. 2017. http://www.who.int/emergencies/mers-cov/risk-assessment-july-2017.pdf?ua=1. Accessed 28 July 2017.

14. Munoz-Price I, et al. SHEA expert guidance: isolation precautions for visitors. Infection control \& hospital epidemiology. 2015. https://shea-online.org/index.php/practice-resources/41-cur rent-guidelines/422-expert-guidance-isolation-precautions-for-visitors. Accessed 2 Aug 2017.

15. Centers for Disease Control and Prevention. Infection prevention and control recommendations for hospitalized patients under investigation (PUIs) for Ebola virus disease. 2015. https://www. cdc.gov/vhf/ebola/healthcare-us/hospitals/infection-control.html. Accessed 4 Feb 2017.

16. Rogers S. Why can't I visit? The ethics of visitation restrictions - lessons learned from SARS. Crit Care. 2004;8(5):300-2.

17. Centers for Disease Control and Prevention. Guidelines for isolation precautions: preventing transmission of infectious agents in healthcare settings. 2007. https://www.cdc. gov/infectioncontrol/guidelines/isolation/prevention.html. Accessed 2 May 2017.

18. Vastag B. Superbug stalked NIH hospital last year, killing six. The Washington Post. 2012. https://www.washingtonpost.com/national/health-science/superbug-stalked-nih-hospital-lastyear-killing-six/2012/08/22/5be18b1a-ec66-11e1-9ddc-340d5efb1e9c_story.html. Accessed 28 July 2017.

19. Centers for Disease Control and Prevention and the Healthcare Infection Control Practices Advisory Committee (HICPAC). Guidelines for environmental infection control in health-care facilities. Morb Mortal Wkly Rep. 2003;52(RR10):1-42.

20. Miller S, et al. Implementing a negative-pressure isolation ward for a surge in airborne infectious patients. Am J Infect Control. 2017;45(6):652-9.

21. Lax S, et al. Bacterial colonization and succession in a newly opened hospital. Sci Transl Med. 2017;9(391) https://doi.org/10.1126/scitranslmed.aah6500.

22. Centers for Disease Control and Prevention. SARS, infection control in healthcare facilities. 2005. https://www.cdc.gov/sars/guidance/i-infection/healthcare.html. Accessed 14 July 2017.

23. Erasmus V, et al. A qualitative exploration of reasons for poor hand hygiene among hospital workers lack of positive role models and of convincing evidence that hand hygiene prevents cross-infection. Infect Control Hosp Epidemiol. 2009;30(5):415-519.

24. Reed D, Kemmerly S. Infection control and prevention: a review of hospital-acquired infections and the economic implications. Ochsner J. 2009;9(1):27-31.

25. Dick A, et al. A decade of investment in infection prevention: a cost-effectiveness analysis. Am J Infect Control. 2015;43(1):4-9.

26. Sun L. Cost to treat Ebola in the U.S.: $\$ 1.16$ million for two patients. The Washington Post. 2014, November 18. https://www.washingtonpost.com/news/post-nation/wp/2014/11/18/cost- 
to-treat-ebola-in-the-u-s-1-16-million-for-2-patients/?utm_term=.e6a9091e93d8. Accessed 29 Jan 2017.

27. Hayden E. Biodefense since 9/11: the price of protection. Nature. 2011; 477:150-152. http:// www.nature.com/news/2011/110907/full/477150a.html. Accessed 30 July 2017.

28. Association for Professionals in Infection Control and Epidemiology - APIC. Ebola preparedness one year later: a poll of APIC members. 2015. https://apic.org/Resource_/ TinyMceFileManager/Topic-specific/APIC_Ebola_Survey_Results_November_2015.pdf. Accessed 15 Aug 2017.

29. Blue Ribbon Study Panel on Biodefense. Recommending and promoting changes to U.S. policy and law to strengthen national biodefense while optimizing resource investments. 2015. http:// www.biodefensestudy.org/. Accessed 8 July 2017.

30. Bloomfield SF, et al. The effectiveness of hand hygiene procedures in reducing the risks of infections in home and community settings including handwashing and alcohol-based hand sanitizers. Am J Infect Control. 2007;35(10):S27-64.

31. Boyce J, Pittet D. Guidelines for hand hygiene in health-care settings: recommendations of the healthcare infection control practices advisory committee and the HICPAC/SHEA/APIC/IDSA hand hygiene task force. Am J Infect Control. 2002;30(8):S1-46.

32. Grayson ML, Jarvie LJ, Martin R, Johnson PD, Jodoin ME, McMullan C, et al. Significant reductions in methicillin-resistant Staphylococcus aureus bacteraemia and clinical isolates associated with a multisite, hand hygiene culture-change program and subsequent successful statewide roll-out. Med J Aust. 2008;188(11):633-40.

33. Johns Hopkins University Center for Health Security. Health sector resilience checklist for high-consequence infectious diseases - informed by the domestic US Ebola response. 2017. http://www.centerforhealthsecurity.org/our-work/pubs_archive/pubs-pdfs/2017/HCID_Final_ Report_05.23.2017.pdf. Accessed 22 June 2017.

34. Occupational Safety and Health Administration. Ebola: control and prevention. https://www. osha.gov/SLTC/ebola/control_prevention.html. Accessed 8 July 2017.

35. Quinlan B, Loughrey S, Nicklin W, Roth V. Restrictive visitor policies: feedback from healthcare workers, patients, and families. Hosp Q. 2003;7(1):33-7.

36. Rutala W, Weber D. Disinfection and sterilization: an overview. AJIC. 2013;41(5):S2-5.

37. World Health Organization. Standard precautions in healthcare. 2007. http://www.who.int/csr/ resources/publications/EPR_AM2_E7.pdf?ua=1. Accessed 19 May 2017. 